\title{
Editorial
}

\section{Mechanisms of mitochondrial membrane permeabilization}

\author{
JC Reed ${ }^{1}$ and G Kroemer ${ }^{\star 2}$ \\ 1 The Burnham Institute, La Jolla, California, CA 92037, USA \\ 2 Institute Gustave Roussy, 94805 Villejuif, France \\ * Corresponding author: G Kroemer, Research Director CNRS-UMR1599, \\ Institute Gustave Roussy, 94805 Villejuif, France \\ E-mail: kroemer@pobox.igr.fr
}

This issue of Cell Death and Differentiation contains six review articles ${ }^{1-6}$ devoted to the topic of mitochondria and their role in apoptosis. The reviews are provided by some of the world's leading groups working on this important subject.

An incontrovertable role for mitochondria in inducing apoptosis and controlling cell death commitment has been established. However, for the past several years, controversy has abounded as to the precise mechanisms by which mitochondria control cell life/death decisions.

Release of cytochrome $c$ from mitochondria couples these organelles to a pathway for caspase activation and apoptosis induction. However, inhibiting caspases and preventing apoptosis does not necessarily protect cells from undergoing cell death via a non-apoptotic mechanism following cytochrome $c$ release. This observation raises the question: What is the cell death commitment mechanism? Theories abound as to why cells sometimes still die even when caspases are inhibited, and several of the competing ideas are reflected in the reviews provided in this issue.

Another subject given considerable attention in the review articles is the question of how cytochrome $c$ escapes from mitochondria, and in particular, how Bcl-2 family proteins regulate this event. Several different models have been proposed, some involving swelling and rupture of mitochondria due to problems with inner membrane permeability barrier function and others envisioning selective loss of outer membrane barrier function without irreversible damage to these organelles. These different points of view are also articulated in the reviews provided. As will become clear to the reader, the precise details of the cytochrome $c$ release mechanism remain controversial, even within the swelling pro and con camps. Thus, nearly as many opinions are voiced as there are articles on the subject.
Also found within the review articles in this issue is discussion of the role of $\mathrm{Bcl}-2$ family proteins as pore-like molecules, and competing theories about their mechanisms are entertained.

Why has so much controversy haunted the field? Partly, the problem may be explained by the technical difficulties of studying mitochondria and the imprecise tools (probes) available for monitoring the status of these organelles their respiratory activity, balances and imbalances of ionfluxes, and integrity of membranes. In this regard, one of the reviews discusses a new technique for local monitoring of $\mathrm{pH}$, using $\mathrm{pH}$-sensitive mutants of the Green Fluorescent Protein (GFP), and other contributions in this issue illustrate the diversity of traditional methods that can be brought to task for studying mechanism of mitochondrial participation in apoptosis and cell death. However, it seems probable that at least some of the present controversy that characterizes the field of apoptosis derives from the likelihood that more than one mechanism can result in events such as release of cytochrome $c$ or mitochondrial membrane depolarization. Thus, it could be argued that everyone's model is correct, but only in certain cellular contexts and scenarios. Regardless, we hope that apoptosis researchers will find the review articles contained in this issue informative, enlightening, and stimulating. May the ideas shared by the authors encourage further debate, and above all, further experimentation.

\section{References}

1. Vieira HLA, Haouzi D, El Hamel C, Jacotot E, Belzacq A-S, Brenner C and Kroemer G (2000) Permeabilization of the mitochondrial inner membrane during apoptosis. Impact of the adenine nucleotide translocator. Cell Death Differ. 7: $1146-1154$

2. Matsuyama S and Reed JC (2000) Mitochondria-dependent apoptosis and cellular pH regulation. Cell Death Differ. 7: 1155-1165

3. Korsmeyer SJ, Wei MC, Saito M, Weiler S, Oh KJ and Schlesinger P (2000) Proapoptotic cascade activates BID, which oligomerizes BAK or BAX into pores that result in the release of cytochrome c. Cell Death Differ. 7: 1166-1173

4. Tsujimoto $Y$ and Shimizu S (2000) VDAC regulation by the Bcl-2 family of proteins. Cell Death Differ. 7: 1174-1181

5. Harris MH and Thompson $\mathrm{CB}$ (2000) The role of the Bcl-2 family in the regulation of outer mitochondrial membrane permeability. Cell Death Differ. 7: 1182-1191

6. Von Ahsen O, Waterhouse NJ, Kuwana T, Newmeyer DD and Green DR (2000) The 'harmless' release of cytochrome c. Cell Death Differ. 7: 1192-1199 